\section{Christoph Bachmann}

\section{Evidence-Based Medicine}

Mitte der Neunzigerjahre des letzten Jahrhunderts tauchte in diesem Zusammenhang erstmals der Begriff «Evidence-Based Medicine» (Evidenzbasierte Medizin) auf. Geprägt wurde der Ausdruck vom Forscherteam um David Sackett, der im Department of Clinical Epidemiology and Biostatistics an der McMaster University im kanadischen Hamilton forschte. 1996 publizierten Sackett et al. [1] im British Medical Journal einen wegweisenden Artikel mit dem Titel «Evidence-Based Medicine: What It Is and What It Isn't». Der Kernsatz dieser Publikation lautet: "Evidence-based medicine is the conscientious, explicit, and judicious use of current best evidence in making decisions about the care of individual patients.»

Die deutsche Übersetzung davon lautet: «Evidenzbasierte Medizin ist der gewissenhafte, ausdrückliche und umsichtige Gebrauch der aktuell besten Beweise für Entscheidungen in der Versorgung eines individuellen Patienten.»

Diese «aktuell besten Beweise» stützen sich demnach auf Publikationen, die Studien vorstellen und eine Therapiemassnahme als wirksam, teilweise wirksam oder unwirksam beschreiben.

Es gibt Organisationen, die der Ärzteschaft und anderen interessierten Fachpersonen Sekundärliteratur zur Verfügung stellen, in der systematische Evidenzrecherchen und -bewertungen vorgestellt werden, d.h. anhand der ausgewerteten Primärliteratur die Evidenz einer Behandlung bestimmt wird [2,3]. Ein bekanntes Qualitätsmessinstrument ist der Jadad-Score, der die formale Qualität einer Studie beurteilt und nicht

\title{
Evidenzbasierte pflanzliche Arzneimittel in der Schweiz
}

Entgegen der in weiten Kreisen der Medizin verbreiteten Meinung gibt es viele pflanzliche Arzneimittel, deren Wirksamkeit und Sicherheit mit zahlreichen klinischen Studien dokumentiert sind, die dem heutigen Standard der klinischen Forschung entsprechen. Solche Phytotherapeutika gehören also zur Evidence-Based Medicine (EBM). Der folgende Beitrag gibt eine Übersicht über Schweizer Phytotherapeutika mit einem hohen EBM-Level.

die ermittelten Resultate und Schlussfolgerungen.

Ein weiteres wichtiges Instrument zur Evidenzbeurteilung von Behandlungsmethoden sind die sogenannten Meta-Analysen. Dabei handelt es sich um eine nachträgliche systematische Auswertung der publizierten Studien über eine bestimmte Behandlungsmethode. Darin einbezogen werden nur Studien mit einer genügend hohen formalen Qualität. Nach Möglichkeit fassen Meta-Analysen bei vergleichbarem Studiendesign und vergleichbaren Zielvariablen die Daten von überprüften Studien zusammen. Sehr häufig findet in Meta-Analysen der sogenannte «Forest plot», eine grafische Darstellung der Resultate, Verwendung.

Die bekannteste Organisation, die solche Evidenzbewertungen macht, ist die Cochrane Collaboration, eine weltweite Organisation von Ärzten und Wissenschaftlern, die systematische Übersichtsarbeiten zur Bewertung medizinischer Therapien erstellt. Ihre regelmässig publizierten Cochrane Reviews geniessen in der
Medizin höchstes Ansehen. Auch das Logo der Cochrane Collaboration (www.cochrane.org) zeigt einen Forest plot.

\section{Evidenzklassen}

Zur Beurteilung des Evidenzgrades einer Therapiemassnahme, z.B. eines Medikaments, gibt es sogenannte Evidenzklassen oder Evidenzlevel (Tab. 1).

Das Ärztliche Zentrum für Qualität in der Medizin (ÄZQ), eine gemeinsame Einrichtung der deutschen Bundesärztekammer und der Kassenärztlichen Bundesvereinigung (KVB), hat ein eigenes Klassifikationszentrum zur Bezeichnung der Evidenzlevel geschaffen (Tab. 2).

\section{Die Beurteilung pflanzlicher Arzneimittel}

Leider beurteilt die Mehrheit der Ärzteschaft und der Wissenschaftler die Evidenz von pflanzlichen Arzneimitteln negativ. Phytotherapeutika werden pauschal als nicht wirksam

Tab. 1. Evidenzklassen nach AHCPR [2]

\begin{tabular}{|c|c|}
\hline Stufe Ia & $\begin{array}{l}\text { Wenigstens eine Metaanalyse auf der Basis methodisch hochwertiger randomisierter } \\
\text { kontrollierter Studien (RCT) }\end{array}$ \\
\hline Stufe Ib & Wenigstens eine ausreichend grosse, methodisch hochwertige RCT \\
\hline Stufe IIa & Wenigstens eine hochwertige Studie ohne Randomisierung \\
\hline Stufe IIb & Wenigstens eine hochwertige Studie eines anderen Typs, quasi-experimentelle Studie \\
\hline Stufe III & $\begin{array}{l}\text { Mehr als eine methodisch hochwertige nichtexperimentelle Studie wie etwa } \\
\text { Vergleichsstudien, Korrelationsstudien oder Fall-Kontroll-Studien }\end{array}$ \\
\hline Stufe IV & $\begin{array}{l}\text { Meinungen und Überzeugungen von angesehenen Autoritäten (aus klinischer } \\
\text { Erfahrung); Expertenkommissionen; beschreibende Studien }\end{array}$ \\
\hline Stufe V & Fallserie oder eine oder mehrere Expertenmeinungen \\
\hline
\end{tabular}

(๑) 2014 S. Karger GmbH, Freiburg
Dr. Christoph Bachmann

Hirschmattstrasse 46, 6003 Luzern, Schweiz

c.a.bachmann@bluewin.ch 


\section{Die Evidenz von Schweizer Phytotherapeutika}

In seinem Referat «Evidence-based Medicine - eine Hilfestellung für phytotherapeutisch tätige Ärzte und Apotheker» [5], gehalten an der 28. Schweizer Jahrestagung für Phytotherapie» vom 21.11.2013 in Baden, stellte PD Dr. med. Andreas Schapowal den Evidenzlevel verschiedener pflanzlicher Präparate vor, die in der Schweiz hergestellt werden und im Handel sind (Tab. 3). Dabei verwendete er den oben genannten Evidenzlevel des ÄZQ.

Schapowal beurteilte den Evidenzlevel der in Tabelle 3 aufgelisteten Präparate anhand der zur Verfügung stehenden Literatur. Als Beispiel dafür dienen hier die Beurteilungen von Ze 117 sowie von Ze 339.

\section{Ze 117}

Von diesem Johanniskraut-Extrakt existieren zwei positive Meta-Analysen, wovon die eine von der Cochrane Collaboration 2005 als CochraneReview publiziert wurde [6]. Weiter gibt es 18 CRT(controlled randomised trial)-Studien mit Ze 117. Hierbei ist vor allem eine randomisierte, placebokontrollierte Doppelblindstudie mit 150 Patienten zu erwähnen [7]. Ze 117 wurde auch in randomisierten, doppelblinden Vergleichsstudien gegen etablierte Antidepressiva geprüft einerseits gegen Fluoxetin [8], andererseits gegen Imipramin [9]. In beiden Fällen erwies sich Ze 117 gegenüber Placebo als hochsignifikant überlegen und Fluoxetin und Imipramin gegenüber als gleichwertig sowie überlegen in Bezug auf die Verträglichkeit. Diese Studienlage reicht aus, um Ze 117 den Evidenzlevel 1 zu verleihen.

\section{Ze 339}

Bei diesem Pestwurz-Extrakt zitierte Schapowal vier randomisierte, kontrollierte Doppelblindstudien [1013]. Drei dieser Studien sind Vergleichsstudien, und zwar gegen Cetirizin [10], gegen Fexofenadin [12] sowie 
gegen Desloratadin [13]. Als Dosisfindungsstudie wurde Ze 339 auch gegen Placebo überprüft [11]. Da bei diesen Studien eine systematische Übersichtsarbeit fehlt, ordnete Schapowal Ze 339 den Evidenzlevel 2 zu.

\section{Weitere Schweizer \\ Phytotherapeutika}

Schapowal hat eine Auswahl derjenigen Schweizer Phytotherapeutika getroffen, die auch in der Schweiz hergestellt werden. Es gibt aber auf dem Schweizer Markt eine Reihe weiterer pflanzlicher Arzneimittel mit einem hohen Evidenzlevel (Tab. 4).

\section{$E G b^{\circledR} 761$}

EGb 761 ist einer der am besten untersuchten Pflanzenextrakte und wirkt gegen kognitive Störungen. Es gibt viele verschiedene CRT-Studien sowie placebokontrollierte und Vergleichsstudien (z.B. [14, 15]). Eine Meta-Analyse liegt nicht vor. Evidenzlevel $1 \mathrm{~b}$.

\section{$E P s^{\circledR} 7630$}

Dieser standardisierte Extrakt aus Pelargonium sidoides wird gegen akute Bronchitis verschrieben. Es gibt randomisierte, placebokontrollierte doppelblinde Studien sowie eine Meta-Analyse der Cochrane Collaboration [16]. Evidenzlevel Ia.

\section{$W S^{\circledR} 5570$}

Der Johanniskraut-Extrakt WS 5570 zählt ebenso zu den Pflanzenextrak- ten, über die Dutzende von Publikationen erschienen sind. Es gibt eine Reihe von randomisierten, placebokontrollierten doppelblinden Studien (z.B. [17]), jedoch keine Meta-Analyse. Evidenzlevel Ib.

\section{PRO 160/120}

Bei PRO 160/120 handelt es sich um ein Kombinationspräparat aus Serenoa repens und Urtica dioica (160 mg Sägepalmen-Extrakt (WS ${ }^{\circledR}$ 1473) und $120 \mathrm{mg}$ Brennnessel-Extrakt (WS $\left.{ }^{\circledR} 1031\right)$ ). Über PRO 160/120 wurden randomisierte, placebokontrollierte doppelblinde Studien sowie Vergleichsstudien (z.B. $[18,19])$ publiziert. Eine Meta-Analyse fehlt. Evidenzlevel Ib.

Extrakt aus Echinacea purpurea

Bei diesem Extrakt handelt es sich um eine standardisierte Zubereitung aus den oberirdischen Teilen und aus der Wurzel von E. purpurea. Verschiedene randomisierte, placebokontrollierte Doppelblindstudien mit Positivresultaten wurden über diesen Extrakt publiziert (z.B. [20, 21]). Eine Meta-A nalyse liegt nicht vor. Evidenzlevel Ib.

\section{160}

Dieser standardisierte Johanniskraut-Extrakt wurde mehrfach in randomisierten, doppelblinden Studien gegenüber Placebo überprüft (z.B. [22]). Eine Meta-Analyse, die nur mit LI 160 durchgeführt wurde, gibt es nicht. Evidenzlevel Ib.

Tab. 4. Evidenzlevel weiterer Schweizer Phytotherapeutika gemäss AHCPR

\begin{tabular}{|c|c|c|c|}
\hline Extrakt & Arzneipflanze & Anwendung & Evidenzlevel \\
\hline $\mathrm{EGb}^{\circledR} 761$ & Ginkgo biloba & kognitive Störungen & $\mathrm{Ib}$ \\
\hline $\mathrm{EPs}^{\circledR} 7630$ & Pelargonium sidoides & Bronchitis & Ia \\
\hline $\mathrm{WS}^{\circledR} 5570$ & Hypericum perforatum & $\begin{array}{l}\text { leichte bis mittelschwere } \\
\text { Depression }\end{array}$ & $\mathrm{Ib}$ \\
\hline PRO 160/120 & $\begin{array}{l}\text { Serenoa repens, } \\
\text { Urtica dioica }\end{array}$ & $\begin{array}{l}\text { benigne Prostatahyperplasie } \\
(\mathrm{BPH})\end{array}$ & $\mathrm{Ib}$ \\
\hline STW3-VI & H. perforatum & $\begin{array}{l}\text { leichte bis mittelschwere } \\
\text { Depressionen }\end{array}$ & $\mathrm{Ib}$ \\
\hline $\begin{array}{l}\text { Echinacea-Extrakt aus } \\
\text { Kraut und Wurzel von } \\
\text { Echinacea purpurea }\end{array}$ & E. purpurea & grippale Infekte & $\mathrm{Ib}$ \\
\hline LI 160 & H. perforatum & $\begin{array}{l}\text { Verstimmungszustände, } \\
\text { Stimmungsschwankungen }\end{array}$ & $\mathrm{Ib}$ \\
\hline
\end{tabular}

\section{1370}

Auch der Extrakt LI 1370 aus Ginkgo biloba wurde in randomisierten, doppelblinden Studien mit Placebo verglichen (z.B. [23]). Eine Meta-Analyse gibt es nicht. Evidenzlevel Ib.

\section{Fazit}

Diese Auflistung von Schweizer Phytotherapeutika mit einem hohen Evidenzlevel erhebt nicht den Anspruch auf Vollständigkeit! Es gibt noch weitere in der Schweiz erhältliche Phytotherapeutika, die einen hohen Evidenzlevel aufweisen!

\section{Vergleichsstudien}

Es gibt eine Reihe von Vergleichsstudien, in denen pflanzliche Arzneimittel der Schweiz mit einem synthetischen Standard verglichen wurden. Hier eine Auswahl davon:

\section{WS 5570 mit Paroxetin}

Der Hypericum-Extrakt WS 5570 ist mehrfach mit Serotonin-Wiederaufnahmehemmern (SSRI) verglichen worden. Anghelescu et al. [24] bewiesen, dass WS 5570 in der Folgebehandlung einer moderaten bis schweren Depression mit Paroxetin ebenbürtig ist.

\section{Ze 117 mit Fluoxetin}

Auch der Johanniskraut-Extrakt Ze 117 ist mit einem SSRI geprüft worden. Schrader [8] wies im Jahr 2000 nach, dass Ze 117 in der Behandlung von leichten bis mittleren Depressionen ebenso wirksam ist wie Fluoxetin. In Bezug auf die Wirksamkeit ist das pflanzliche Präparat dem synthetischen Wirkstoff überlegen.

\section{STW3-VI mit SSRI}

Auch von diesem JohanniskrautExtrakt gibt es eine Vergleichsstudie mit SSRI aus dem Jahr 2012 [25]. Bei dieser Versorgungsstudie wurde der Hypericum-ExtraktSTW3-VI im Praxisalltag mit SSRI, vor allem mit Citalopram, verglichen. Auch hier konnte die gleiche Wirksamkeit des 
pflanzlichen Präparats und eine bessere Verträglichkeit festgestellt werden.

\section{PRO 160/120 mit Tamsulosin}

Das Serenoa-Urtica-Kombinationspräparat PRO 160/120 ist 2006 von Engelmann et al. [19] mit dem BPHStandard Tamsusolin verglichen worden. Bei der Veränderung des International Prostate Symptom Score (I-PSS) erwies sich Pro 160/120 als gleich wirksam wie Tamsulosin. Auch hier zeigte sich eine bessere Verträglichkeit.

\section{160 mit Sertralin}

Die oben erwähnte Studie von Sarris et al. [22] wurde dreiarmig angelegt. LI 160 wurde mit Placebo und Sertralin verglichen und kann hier also auch als Vergleichsstudie erwähnt werden. LI 160 erwies sich gegenüber Sertralin als nicht unterlegen.

\section{Grundversicherung}

Die hohe Qualität der Studienlage der hier erwähnten Schweizer Phytotherapeutika hat dazu geführt, dass alle in die Spezialitätenliste aufgenommen wurden. Sie gehören also zur obligatorischen Grundversicherung und werden von dieser erstattet, wenn sie ärztlich verschrieben werden. Eingang in diese Liste erhalten nur Arzneimittel mit einem entsprechenden klinischen Wirksamkeitsnachweis. Die vollständige Liste findet man unter $h t t p: / /$ bag.e-mediat.net.

\section{Schlussfolgerung}

Entgegen der leider in weiten Kreisen der Medizin verbreiteten Meinung gibt es viele pflanzliche Arzneimittel, deren Wirksamkeit und Sicherheit mit zahlreichen klinischen Studien nachgewiesen wurde. In der Schweiz ist eine Reihe von Phytotherapeutika auf dem Markt, die einen hohen Evidenzlevel aufweisen. Dies kann sowohl mit der deutschen Evidenz-
level-Einteilung des ÄZQ als auch mit der Einteilung gemäss der amerikanischen Agency for Health Care Policy and Research (AHCPR) gezeigt werden. Die hier aufgelisteten pflanzlichen Arzneimittel entsprechen meistens dem zweithöchsten Evidenzlevel, in einzelnen Fällen sogar dem höchsten.

Weiter gibt es verschiedene Vergleichsstudien, in denen ein in der Schweiz erhältliches Phytotherapeutikum mit einem synthetischen Standard verglichen wurde. Mit diesen Studien konnte jeweils die Nichtunterlegenheit oder sogar die Gleichwertigkeit des pflanzlichen Präparats dokumentiert werden. Weiter zeigten die Resultate auch durchwegs eine bessere Verträglichkeit des pflanzlichen Präparats. Mit diesen Resultaten erweisen sich die entsprechenden Präparate als eine valable therapeutische Alternative gegenüber den synthetischen Arzneimitteln.

\section{Literatur}

1 Sackett DL, Rosenberg WMC, Gray JAM, Haynes RB, Richardson WS: Evidence-based medicine: what it is and what it isn't. BMJ 1996;312:71-72.

2 Agency for Health Care Policy and Research. $w w w$. ahrq.gov.

3 Ärztliches Zentrum für Qualität in der Medizin. www.aezq.de.

4 Tacklind J, MacDonald R, Rutks I, Wilt TJ: Serenoa repens for benign prostatic hyperplasia. Cochrane Database Syst Rev 2009;2:CD001423.

5 Schapowal A: Evidence-based Medicine - eine Hilfestellung für phytotherapeutisch tätige Ärzte und Apotheker. Referat gehalten an der 28. Schweizerischen Tagung für Phytotherapie, 11.11.2013, Kongresszentrum TRAFO, Baden, Schweiz.

6 Linde K, Mulrow CD, Berner M, Egger M: St John's wort for depression. Cochrane Database Syst Rev 2005;2:CD000448.

7 Schrader E, Meier B, Brattstroem A: Hypericum treatment of mild-moderate depression in a placebo-controlled study. A prospective, double-blind, randomized, placebo-controlled, multicenter study. Human Psychopharmacol 1998;13:163-169.

8 Schrader E: Equivalence of St John's wort extract (Ze 117) and fluoxetine: a randomized, controlled study in mild-moderate depression. Internat Clin Psychopharmacol 2000;15:61-68.

9 Woelk H: Comparison of St John's wort and imipramine for treating depression: randomised controlled trial. BMJ 2000;321:536-539.

10 Schapowal A; on behalf of Petasites Study Group: Randomised controlled trial of butterbur and cetirizine for treating seasonal allergic rhinitis. BMJ 2002;324:144-146.

11 Schapowal A; on behalf of Petasites Study Group: Butterbur Ze 339 for the treatment of intermittent allergic rhinitis: dose-dependent efficacy in a prospective, double-blind, placebo-controlled study. Arch Otolaryngol Head Neck Surg 2004;130:13811386.

12 Schapowal A; on behalf of Petasites Study Group: Treating intermittent allergic rhinitis: a prospective, randomized, placebo and antihistamine-controlled study of Butterbur extract Ze 339. Phytother Res 2005;19:530-537.

13 Dumitru A, Shamji M, Wagenmann M, et al: Petasol butenoate complex (Ze 339) relieves allergic rhinitis-induced nasal obstruction more effectively than desloratadine. J Allergy Clin Immunol 2011;127:1515-1521.

14 Napryeyenko O, Borzenko I; GINDEM-NP Study Group: Ginkgo biloba special extract in dementia with neuropsychiatric features. A randomised, placebo-controlled, double-blind trial. Arzneimittelforschung 2007;57:4-11.

15 Bachinskaya N, Hoerr R, Ihl R: Alleviating neuropsychiatric symptoms in dementia: the effects of Ginkgo biloba extract EGb ${ }^{\circledR}$ 761. Findings from a randomized controlled trial. Neuropsychiatr Dis Treatment 2011;7:209-215.

16 Timmer A, Günther J, Rücker G, Motschall E, Antes G, Kern WV: Pelargonium sidoides extract for acute respiratory tract infections. Cochrane Database Syst Rev 2008;3:CD006323.

17 Kasper S, Volz HP, Möller HJ, Dienel A, Kieser M: Continuation and long-term maintenance treatment with Hypericum extract WS ${ }^{\circledR} 5570$ after recovery from an acute episode of moderate depression - a double-blind, randomized, placebo controlled long-term trial. Eur Neuropsychopharmacol 2008;18:803-813.

18 Lopatkin N, Sivkov A, Walther C, et al: Long-term efficacy and safety of a combination of sabal and urtica extract for lower urinary tract symptoms a placebo-controlled, double-blind multicenter trial. World J Urol 2005;23:139-146.

19 Engelmann U, Walther C, Bondarenko B, Funk P, Schläfke S: Efficacy and safety of a combination of sabal and urtica extract in lower urinary tract symptoms. A randomized, double-blind study versus tamsulosin. Arzneimittelforschung 2006; 56:222-229.

20 Brinkeborn RM, Shah DV, Degenring FH: Echinaforce and other Echinacea fresh plant preparations in the treatment of the common cold. A randomized, placebo controlled, double-blind clinical trial. Phytomedicine 1999;6:1-6.

21 Jawad M, Schoop R, Suter A, et al: Safety and efficacy profile of Echinacea purpurea to prevent common cold episodes: a randomized, double-blind, placebo-controlled trial. Evid Based Complement Alternat Med 2012;2012:841315

22 Sarris J, Fava M, Schweitzer I, Mischoulon D: St John's wort (Hypericum perforatum) versus sertraline and placebo in major depressive disorder: continuation data from a 26-week RCT. Pharmacopsychiatry 2012;45:275-278.

23 Trick L, Boyle J, Hindmarch I: The effects of Ginkgo biloba extract (LI 1370) supplementation and discontinuation on activities of daily living and mood in free living older volunteers. Phytother Res 2004:18:531-537.

24 Anghelescu IG, Kohnen R, Szegedi A, Klement S, Kieser M: Comparison of Hypericum extract WS ${ }^{\circledR}$ 5570 and paroxetine in ongoing treatment after recovery from an episode of moderate to severe depression: results from a randomized multicenter study. Pharmacopsychiatry 2006;39:213-219.

25 Kresimon J, Kiencke P, Viehmann K, Kolb C, Müller J, Rychlik R: Versorgung von Patienten mit mittelschwerer Depression unter Therapie mit Hypericum-Extrakt STW3-VI im Vergleich zu selektiven Serotonin-Wiederaufnahmehemmern (SSRI) im Praxisalltag. Gesund Ökon Qual Manag 2012;17:198-205. 